\title{
Responsabilité sociale et accréditation. Une nouvelle frontière pour l'institution de formation
}

\author{
Social accountability and accreditation. \\ A new frontier for educational institutions
}

\author{
Charles BOELEN ${ }^{1}$, Paul GRAND'MAISON ${ }^{2}$, Joël LADNER ${ }^{3}$ et Dominique PESTIAUX ${ }^{4}$ au \\ nom du groupe d'action prioritaire "Santé et Société » de la Société internationale francophone \\ d'éducation médicale (SIFEM) \\ 1 Consultant international, ancien coordonnateur du programme de l'OMS (Genève) des ressources humaines pour la \\ santé \\ 2 Faculté de médecine et des sciences de la santé, Université de Sherbrooke, Canada \\ 3 Faculté de médecine, Université de Rouen, France \\ 4 Faculté de médecine, Université Catholique de Louvain, Belgique
}

Manuscrit reçu le 30 septembre 2008; commentaires éditoriaux formulés aux auteurs le 8 octobre 2008 ; accepté pour publication le 13 octobre 2008

\author{
Mots clés : \\ Besoins de société ; \\ système de santé ; \\ responsabilité sociale ; \\ excellence de \\ l'institution de forma- \\ tion; \\ éducation médicale ; \\ normes ; \\ accréditation
}

\section{Keywords:}

Social needs; health system; social responsibility; social accountability; excellence of educational institution; medical education; norms; accreditation
Résumé - Contexte : Être socialement responsable, c'est être comptable devant la société des actions que l'on entend mener en sa faveur. En matière de santé, la responsabilité sociale est l'engagement à répondre de façon aussi performante que possible aux besoins de santé prioritaires du citoyen et de la société, notamment en érigeant comme principes fondateurs les valeurs de qualité, équité, pertinence et efficience, et la participation active à l'évolution du système de santé. Exégèse : Pour l'institution de formation, la responsabilité sociale devrait être appréciée à l'aide de normes relevant de trois domaines interdépendants : la conception, la production et l'utilisabilité des personnels de santé. L'institution qui forme et qui vérifie son impact sur la société mérite un label d'excellence, en ce qu'elle assume pleinement son rôle d'acteur responsable dans le système de santé et se pose en garante de l'intérêt général. Conclusion : Dans un monde plus enclin à la recherche d'efficience et de transparence, cette nouvelle posture de l'institution de formation s'imposera à l'avenir. Un cadre général de normes illustrant la responsabilité sociale est proposé pour une réactualisation de l'évaluation et l'accréditation.

Abstract - Background: Being socially responsible implies accountability to society for actions intended to serve it. In the health field, social accountability is a commitment to respond as best as possible to priority health needs of citizens and society, namely by meeting basic principles of quality, equity, relevance and effectiveness, and by active participation in health system development. Exegesis: For an educational institution, social accountability should be measured by sets of norms relative to three interdependent domains: conceptualization, production and utilisability of health personnel. An educational institution that does verify its impact on society deserves a label of excellence for it fully assumes the position of responsible partner in the health system and is dedicated to public interest. Conclusion: In a world increasingly incline to value performance and transparency, this posture of the educational institution will become a rule in the future. A general framework of norms is proposed to revisit current evaluation and accreditation practices. 
Et si on reconnaissait l'excellence de l'institution de formation des personnels de santé au fait que les diplômés possèdent toutes les compétences voulues et sont en mesure de les appliquer dans leur pratique professionnelle? L'institution de formation sera-t-elle un jour tenue pour partie responsable de la façon dont seront utilisés ses diplômés ? Probablement, car l'institution de formation est de plus en plus incitée à fonctionner sur le mode d'une entreprise performante, c'est-à-dire, à être plus explicite sur ses objectifs et plus comptable de ses réalisations au bénéfice de la société. Nul doute que des politiques nationales en matière d'enseignement supérieur et de santé publique s'engageront dans cette voie. Encore faut-il qu'il existe une véritable volonté politique, une cohérence entre l'identification des besoins de santé d'une population, le fonctionnement d'un système de santé et la stratégie de formation. Quant à l'institution de formation, elle devra disposer d'autonomie et de moyens lui permettant de mettre mieux à profit son potentiel d'innovation pour relever ces défis.

\section{Cela va mieux et pourtant, c'est la crise!}

Depuis les années 50, la qualité de la formation des professionnels de santé s'est progressivement améliorée au gré d'avancées telles que : la planification des programmes d'éducation par objectifs, l'apprentissage par problèmes, la formation en équipe multiprofessionnelle, l'immersion précoce dans la communauté et les services de santé de première ligne, une formation centrée sur les besoins de l'apprenant, la formation pédagogique des enseignants, la recherche en pédagogie et plus récemment l'usage de l'informatique et d'internet. De plus, les normes utilisées pour l'évaluation de la qualité et l'accréditation des institutions tendent à tenir compte de ces développements.

Ceci serait pour nous rassurer si on ne constatait que ces efforts, tous louables en soi, n'ont pas eu raison de la crise que traverse depuis plusieurs décennies le développement des personnels de santé à travers le monde. Jugeons-en par l'inventaire accablant suivant : déficit quantitatif en personnels de santé, inadéquation du nombre des spécialités au regard des besoins de santé, manque chronique de cadres destinés aux soins de santé primaires, migration des professionnels de santé vers des zones de plus grande attractivité financière et sociale, démédicalisation des régions rurales, manque général de compétences et de volonté des décideurs en prévention et promotion de la santé, timide mobilisation du citoyen à assumer sa part de responsabilité pour le maintien de sa propre santé, absence d'incitation à travailler en partenariat avec le secteur social pour agir plus efficacement sur les déterminants sociaux de la santé, dérive vers une marchandisation des services au dépends de l'idéalisme professionnel, perte d'autorité des professions de santé face à l'administration et au public, démotivation des professions de santé.

Comment l'institution (convenons d'utiliser le terme générique «institution » dans la suite de l'article pour désigner universités, facultés ou écoles) peut-elle contribuer à atténuer cette crise, dans l'exercice de ses missions de formation, de recherche et de service? A-t-elle quelque prise sur les causes de la crise? La conception, la mise en œuvre et le suivi des programmes de formation devraient être établis en fonction des besoins prioritaires de la société et des citoyens et en étroite cohérence avec le processus de développement sanitaire d'un pays. Aussi, dans la mesure où la politique de santé a une incidence sur l'éventail des compétences que devront maîtriser les futurs professionnels de la santé, l'institution devrait s'y intéresser, voire participer à son élaboration et sa mise en œuvre. Cette posture volontariste de l'institution devrait être clairement affirmée dans l'énoncé de sa mission et de ses objectifs institutionnels. De plus, son plan de développement stratégique devrait être élaboré en tenant compte de perspectives d'évolution du système de santé et de projections de besoins en matière de personnels de santé, tant sur un plan qualitatif que quantitatif. Et, bien entendu, ses programmes de formation seraient réajustés en conséquence. 
Certes, l'effort d'anticipation et d'adaptation, recommandé à l'institution, est également recommandé aux autres acteurs de santé (pouvoir public, dispensateur de soins, organisme assureur, association professionnelle, ...) car la résolution de la crise des personnels de santé, ou du moins son atténuation, suppose en effet que chacun soit animé d'un même sentiment de responsabilité sociale et soit comptable de ses réalisations devant la société.

\section{Ce que la responsabilité sociale implique}

Dans le cas des facultés de médecine, l'Organisation mondiale de la santé (OMS) définit leur responsabilité sociale comme étant «l'obligation d'orienter la formation qu'elles donnent, les recherches qu'elles poursuivent et les services qu'elles dispensent, vers les principaux problèmes de santé de la communauté, région ou/et nation qu'elles ont comme mandat de servir. Les principaux problèmes de santé seront identifiés conjointement par les gouvernements, les organismes, les professionnels de la santé et le public ${ }^{[1]}$. Ainsi, deux dimensions émergent : altruisme et altérité ; altruisme, par l'intérêt prioritaire porté au bien-être de la société, et altérité, par le souci d'intégration au contexte social. On peut en déduire deux groupes de principes susceptibles de servir de référence : principes humanistes, relatifs à la protection des personnes, et principes systémiques, relatifs au rapport de l'institution au système de santé.

\section{Principes humanistes}

Quatre principes sont retenus pour illustrer le droit des personnes à la santé, tant sur le plan individuel que collectif : la qualité, l'équité, la pertinence et l'efficience. Le principe de qualité garantit au citoyen la jouissance des meilleures mesures possibles pour protéger, rétablir et promouvoir son état de bien-être physique, mental et social, notamment par un éventail de services conformes au confort et à la dignité de la personne. Le principe d'équité tend à assurer à chaque citoyen plein accès aux services de santé, à l'abri de toute forme de discrimination. Le principe de pertinence incite à répondre aux besoins de santé prioritaires et à porter une attention particulière aux personnes et groupes les plus vulnérables de la société. Enfin, le principe d'efficience encourage le meilleur usage des ressources de santé, humaines et matérielles, pour servir l'intérêt général de façon aussi performante que possible.

\section{Principes systémiques}

Ces principes se rapportent à la compréhension de la complexité du système de santé et à la capacité d'y trouver une place aussi utile que possible. L'institution mènera une action d'autant plus efficace qu'elle le fera en concertation avec d'autres partenaires du système de santé, notamment, les décideurs politiques, les gestionnaires de la santé, les professions de santé et la société civile. Dans la réalisation des principes humanistes énoncés plus haut, chacun a son rôle à jouer qui doit être connu des autres. Ainsi, le décideur politique proposerait une vision à long terme d'un système de santé, cohérent et fédérateur, le gestionnaire allouerait des ressources pour un fonctionnement du système de santé conforme à cette vision, le professionnel de santé acquerrait des compétences pour offrir des prestations nouvelles, tandis que le citoyen participerait davantage à protéger sa propre santé et celle de la collectivité. Il incombe à chaque acteur de se remettre en question et de s'engager à orienter son action au service de l'intérêt général, tant il est vrai que seule une action conjuguée des acteurs pourra faire évoluer un système de santé, et donc ses ressources humaines, vers l'optimisation $^{[2]}$.

La responsabilité sociale de l'institution ne peut s'assumer pleinement que si elle transgresse les frontières de ses prérogatives traditionnelles, pour s'intéresser au devenir des personnels formés. Elle s'arrogera le droit de questionner si ses produits sont utilisés au mieux de l'intérêt général. Ainsi, sa responsabilité sociale comprend un devoir de traçabilité 
en s'aventurant sur un terrain sur lequel son autorité n'est pas formellement reconnue, celui du fonctionnement du système de santé. Nous proposons qu'un label d'excellence soit attribué à une institution qui, sur la base des principes humanistes et systémiques énoncés plus haut, se fixe comme finalité la recherche d'un impact sur la santé.

\section{Excellence de l'institution de formation : I'impact comme finalité}

En s'interrogeant sur sa raison d'être et la finalité de son action, on s'inscrit dans une certaine logique de régulation économique. À quels besoins des citoyens et de la société les futurs diplômés devraient-ils répondre ? Dans quel système de santé travailleraient-ils le mieux ? Quelles nouvelles compétences devraient-ils acquérir pour devenir davantage des promoteurs de la santé que des techniciens de la maladie? Quelles nouvelles carrières faudrait-il prévoir? Quelles seraient les demandes et les offres pour celles-ci ? Comment les motiver à des pratiques de qualité ? Comment se rendre compte que l'éducation est arrivée à ses fins ? Ces questions doivent être posées par l'institution et orienter sa mission. Comme l'institution seule ne peut avoir les réponses, la logique économique veut qu'une relation s'établisse entre l'institution, considérée comme «productrice » de personnels de santé, et les différentes composantes du système de santé, considérées comme « utilisatrices ».

Pour l'institution, l'ambition d'impact commence par la détermination du profil du professionnel de santé à former. Un tel exercice d'anticipation, inspiré d'une analyse prospective des besoins des populations et de l'évolution du système de santé, est indispensable pour donner un sens à l'entreprise de formation. De tels profils, avec leur éventail de compétences, ont été décrits dans des modèles proposés par l'OMS (le médecin cinq étoiles) ${ }^{[3]}$, par le General Medical Council du Royaume Uni (Tomorrow's doctors $)^{[4]}$, ou par le Collège Royal des médecins et chirurgiens du Canada (CanMEDS) ${ }^{[5]}$ ou encore par les tenants du professionnalisme ${ }^{[6]}$.
En même temps, l'institution devra se soucier que ces mêmes compétences seront formellement reconnues et justement valorisées par le système de santé. Trop souvent, l'institution se comporte comme une agence de sous-traitance vis-à-vis du système de santé plutôt qu'en partenaire de plein droit. Or, c'est de cette timidité que l'institution doit s'affranchir car un accord sur un profil doit nécessairement résulter d'une franche concertation avec ceux qui définissent la politique de santé, ceux qui organisent le système de soins, ceux qui créent des postes de travail et soutiennent en définitive les prestations où les compétences enseignées sont requises ${ }^{[7]}$.

Cette concertation est bénéfique si, au sein de l'institution même, l'intérêt général prime sur les intérêts particuliers. Par contre, cet horizon reste éloigné si les titulaires d'une discipline particulière conduisent leurs activités en fonction de leurs propres intérêts, dans l'indifférence d'une perspective sociale plus large. À la faculté de médecine, par exemple, il est commun d'accorder plus de lustre à une découverte en médecine expérimentale qu'à une brillante réussite de programme de santé publique. Chacune de ces réalisations a son importance, mais la prééminence du paradigme bio-médical sur le paradigme socio-économique reste notoire. En matière d'éducation médicale, le même biais existe : trop peu de compétences sont proposées aux futurs médecins pour agir non seulement sur les causes de la maladie mais aussi sur les déterminants sociaux de la santé. À la décharge du chercheur et de l'enseignant, reconnaissons que l'effort d'anticipation et d'imagination pour améliorer l'impact sur la santé de la société est peu valorisé par le leadership académique et les organes de tutelle. Aussi, ne s'étonnerat-on pas si l'essentiel de la créativité en matière d'éducation se reporte sur les contenus du curriculum et les méthodes d'apprentissage.

En somme, beaucoup d'attention sur le processus et pas assez sur la finalité !

Ce constat navrant n'est pas neuf. On peut le faire remonter au début du siècle dernier quand Flexner $^{[8]}$, reconnaissant le déficit d'équité du système de santé américain, recommande que les 
étudiants noirs reçoivent une bonne éducation médicale afin qu'ils contribuent à rehausser le niveau de santé dans leur communauté. Il tente donc cette corrélation entre éducation médicale et santé des populations. C'est en effet un souci d'impact social de l'éducation médicale qui motive son fameux projet d'enquête sur le contenu des programmes de formation dans l'ensemble des facultés de médecine nordaméricaines. Mais cette perspective s'estompa progressivement et au bout du compte la refondation des facultés de médecine se fit, notamment par ses successeurs, sur le seul renforcement du caractère scientifique du curriculum ${ }^{[8]}$. On connaît la suite : le bloc des sciences dures, dites de base, devint le sas préalable à tout contact avec le patient et le milieu social, une introduction retardée et timide des sciences humaines, l'émergence d'une farouche indépendance des disciplines et départements, la verticalisation des enseignements aux dépens d'une intégration plus à même d'aider l'apprenant à appréhender des problèmes de santé complexes et la considération de la santé publique comme une discipline banalisée, voire au rabais. Sans doute, Flexner avaitil bien commencé mais l'affairisme pédagogique a finalement dévoyé son projet de sa finalité sociale. Son modèle est maintenant devenu synonyme d'étanchéité de l'institution vis-à-vis du milieu ambiant. Il reste néanmoins prédominant à travers le monde depuis un siècle et continue à inspirer les paramètres d'accréditation des facultés de médecine. Quel ratage historique d'occasion pour fixer comme finalité à l'institution académique l'impact sur la santé d'une société ${ }^{[9]}$ !

Placer résolument les besoins de santé de la société au centre de ses préoccupations reste proprement révolutionnaire, dans le sens copernicien du terme : l'intérêt général (le soleil) est au centre du dispositif et les intérêts particuliers des différents acteurs de santé (les planètes), parmi lesquels l'institution de formation, gravitent autour. Un tel positionnement doit inciter l'institution à revisiter sa mission, à relativiser sa mission par rapport à celle des autres, à mieux apprécier son potentiel et ses limites, et à tisser des partenariats avec d'autres
Tableau I. Besoins de société et normes.

\begin{tabular}{|c|c|}
\hline Besoins de la société & Normes \\
\hline Rôle de l'institution & Conception du produit \\
\hline Mission de formation & ... Production du produit \\
\hline Satisfaction des besoins & Utilisabilité du produit \\
\hline
\end{tabular}

acteurs pour que ses interventions pèsent davantage et plus durablement sur la santé.

\section{Éventail de normes illustrant la responsabilité sociale}

Le principe de cohérence, établissant une liaison rigoureuse entre les besoins du consommateur, la conception d'un produit, sa fabrication, et sa distribution pour la satisfaction du consommateur, est fondamental pour toute entreprise soucieuse de performance. À sa manière, ce principe s'applique à la notion de responsabilité sociale puisque l'étude des besoins de santé de la société constitue le début d'un processus et la satisfaction de ces besoins son aboutissement. Aussi, proposons-nous que des normes pour l'évaluation et l'accréditation de l'institution de formation s'en inspirent. Elles relèveraient de trois domaines spécifiques, bien que fort interdépendants, à savoir : la conception, la production et l'utilisabilité du «produit », c'est-à-dire, le personnel de santé (tableau I).

Le domaine de la conception explore les références et les engagements sur lesquels l'institution s'appuie pour justifier les personnels de santé à former, ainsi que le mode de gouvernance de l'institution pour tenir un rôle actif dans un système de santé. Le domaine de la production explore les principales composantes relatives à la formation proprement dite, avec un arsenal de normes dont la plupart sont assez proches de celles aujourd'hui utilisées pour l'évaluation et l'accréditation des facultés de médecine. Enfin, le domaine de l'utilisabilité explore les initiatives entreprises par l'institution pour que les 
personnels formés soient utilisés conformément aux engagements formulés à la phase de conception.

Le terme «utilisabilité » est un néologisme choisi à dessein, pour le distinguer de l'idée «d'utilisation » ou «d'utilité » et mettre en évidence le véritable souci de l'institution pour le devenir de ses diplômés. En effet, l'utilisation et l'utilité des personnels formés existent dès lors qu'ils sont intégrés dans une structure de travail et qu'ils donnent satisfaction à l'employeur ou l'usager, sans avoir pour autant une garantie de pratiquer l'ensemble des compétences acquises pendant la formation. Par contre, l' « utilisabilité » renvoie à la notion de concordance entre les compétences acquises et les possibilités de les appliquer sur le terrain. Le domaine de l'utilisabilité comprendra donc des normes illustrant les positions et les démarches de l'institution afin que les personnels formés selon un profil inspiré des besoins de la société puissent trouver un environnement de travail où ce profil est valorisé.

L'institution, appliquant le modèle « conception-production-utilisabilité » (en abrégé : $\mathrm{CPU}$ ), peut se trouver en décalage par rapport à un système de santé qui ne s'acquitterait pas de sa responsabilité sociale avec la même rigueur, par exemple en n'offrant pas ou pas suffisamment de débouchés aux personnels formés en adéquation avec un profil dont l'utilité publique serait pourtant reconnue. L'institution pourrait alors se considérer comme étant en avance sur le système de santé. Reconnaissons que dans bien des cas, c'est l'institution de formation qui est en retrait. Quel que soit le cas, la nécessité de concertation, voire de partenariat durable, entre les différents acteurs de santé est impérative car la responsabilité sociale ne peut être entièrement assumée que si l'ensemble des acteurs tend à partager un même corps de valeurs.

Le tableau II qui suit présente un cadre général à partir duquel des normes, indicateurs et critères spécifiques pourront être définis pour orienter l'institution de formation vers davantage de pertinence sociale. Il comprend trois domaines et 11 sections pour un total de 31 items. On notera qu'un certain degré de redondance existe, probablement inévitable du fait de l'enchaînement rigoureux des éléments constitutifs du modèle.

Une des préoccupations majeures du modèle CPU est le respect de cohérence entre les trois domaines dits de conception, production et utilisabilité. Nous en donnons ci-après quelques illustrations.

- La valeur de l'équité, mentionnée à l'item 1.1, que l'institution déclare être un de ses principes fondateurs, est reprise dans les items de la section 5 (programme de formation) et dans les items 10.1 et 10.2 de la section 10 (emploi).

- L'item 1.3 (système de santé), qui se rapporte à l'intégration à un système de santé orienté vers les besoins du citoyen et de la société, conduit d'abord à un engagement à s'investir dans une zone de santé en partenariat avec d'autres acteurs de santé (items 2.2 et 2.3), ensuite, à l'utilisation de cette zone comme un terrain d'application pour la formation, recherche et la prestation de services (section 4) et, enfin, à une vérification de l'effet de ses actions sur le terrain (items 11.1 et 11.2).

- Le même souci de traçabilité s'observe quand l'institution prend l'engagement de former un type de personnel (voir items 1.4 et 2.4), élabore un programme de formation (item 5.1), procède à l'évaluation des compétences (item 6.3) et vérifie les effets en pratique (item 10.3).

Une illustration de l'utilisation concrète du modèle avec des exemples se rapportant aux différents items devrait faire l'objet d'un autre article.

\section{Rapport avec les systèmes d'évaluation et accréditation existants}

Depuis l'avènement de normes pour évaluer et accréditer les facultés de médecine, il y a quelques décennies, en particulier, en Amérique du Nord avec l'agence du Liaison Committee on Medical Education (LCME), à travers les différentes initiatives nationales qui s'en sont inspirées, et jusqu'à la publication plus récente de standards internationaux par la 
Tableau II. Modèle CPU : conception-production-utilisabilité.

\section{CONCEPTION}

\section{1- Références}

1.1 Valeurs : Référence explicite à un corps de valeurs ( $c$-à- $d$. qualité, équité, pertinence, efficience)

1.2 Population : Référence aux caractéristiques de la population et à ses besoins prioritaires de santé

1.3 Système de santé : Référence à l'évolution du système de santé vers plus de cohérence et d'intégration

1.4 Personnels de santé : Référence aux besoins quantitatifs et qualitatifs. Voir items 1.1, 1.2, 1.3

\section{2- Engagements}

2.1 Mandat : Mission et objectifs institutionnels, conformes aux références

2.2 Terrain : Participation à la gestion de santé d'un territoire et d'une population définie

2.3 Partenariat : Collaboration suivie avec principaux acteurs de la santé, sur le terrain et au plan national

2.4 Produit fini : Définition/ justification du profil du personnel à former (liste de compétences). Voir références

\section{3- Gouvernance}

3.1 Plan stratégique : Ordonnancement des engagements selon un plan largement agréé

3.2 Gestion : Mécanismes de valorisation, coordination et évaluation pour la mise en œuvre du plan

3.3 Ressources : Mobilisation de ressources intérieures et extérieures à la hauteur des engagements

\section{PRODUCTION}

4- Terrain d'application : Formation, recherche et service sur le terrain. Voir item engagement 2.2

\section{5- Programme de formation}

5.1 Objectifs et contenus : Conformes au profil du personnel à former (2.4)

5.2 Déroulement : Exposition précoce, longitudinale et intégrée aux réalités de terrain

5.3 Apprentissage : Participation à la résolution de problèmes de santé complexes, individuels et collectifs

5.4 Stages : Lieux privilégiant les services de santé de première ligne et leur rapport avec d'autres niveaux

6- Étudiants

6.1 Recrutement : Égalité des chances et priorité aux étudiants issus de communautés défavorisées

6.2 Carrière : Orientation et accompagnement pour satisfaire des besoins de santé prioritaires

6.3 Evaluation : Exploration de l'ensemble des compétences figurant dans le profil. Voir item 2.4

7- Enseignants

7.1 Source : Participation d'une variété d'enseignants issus des services sanitaires et sociaux

7.2 Compétences : Enseignants aptes à servir de modèles, en rapport avec le profil à former (item 2.4)

7.3 Soutien : Formation et autres incitatifs pour améliorer santé publique et éducation médicale

8- Recherche : En gestion du système de santé. Voir références (section 1) et utilisabilité (sections 10 et 11)

9- Service : Excellence dans les soins de santé primaires. Voir utilisabilité (sections 10 et 11)

\section{UTILISABILITÉ}

\section{0- Emploi}

10.1 Débouchés : Plaidoyer et collaborations en faveur de professions en besoins prioritaires

10.2 Implantation : Rétention et répartition géographique des diplômés selon besoins. Voir items 1.1 et 1.2

10.3 Prestations : Maintien de compétences des diplômés, en rapport avec le profil. Voir item 2.4

10.4 Pratique : Amélioration des conditions de pratique au niveau de la première ligne. Voir sections 4, 9 et 10

\section{1- Impact}

11.1 Partenariat : Qualité de la relation entre acteurs pour un meilleur fonctionnement du système de santé 11.2 Effets sur la santé : Diminution de risques et promotion de santé sur le terrain. Voir 2.2 et 2.3 et section 4 11.3 Promotion : Diffusion de résultats sur l'utilisabilité auprès des organes décisionnels de la région ou nation 
Tableau III. Besoins de société et normes d'éducation médicale.

\begin{tabular}{|lccc|}
\hline & Conception & Production & Utilisabilité \\
\hline Normes & + & +++ & + \\
actuelles & & & \\
Normes & +++ & +++ & +++ \\
souhaitables & & & \\
\hline
\end{tabular}

Fédération Mondiale d'Éducation Médicale (World federation for medical education - WFME -), l'emphase a été mise dans le domaine de la production, avec un moindre intérêt pour les domaines de conception, et d'utilisabilité ${ }^{[10-12]}$ (tableau III).

Dès 1995, l'OMS, soucieuse que l'éducation médicale soit cohérente avec une politique de santé répondant aux besoins prioritaires de la société, recommanda que la qualité de la formation tienne compte des principes de responsabilité sociale, sans pour autant avoir fait des propositions concrètes en matière de normes, ni avoir pris l'initiative d'encourager explicitement les pays à se doter de systèmes d'accréditation réactualisés ${ }^{[13,14]}$.

Récemment, certaines organisations internationales, telles le réseau The Network Towards Unity for Health, par le biais de son groupe de travail «social accountability and accreditation » ${ }^{[15]}$, la conférence internationale des doyens et des facultés de médecine d'expression française (CIDMEF), par son conseil d'évaluation ${ }^{[16]}$, et la société internationale francophone d'éducation médicale (SIFEM), par son groupe de travail «Santé et société »[17], ont entamé une réflexion conforme à cette orientation. De même, la Foundation for the advancement of international medical education and research (FAIMER), création récente de l'agence américaine Educational commission for foreign medical graduates (ECFMG), dont le principal mandat est de promouvoir l'expertise en éducation médicale au niveau mondial, met en évidence la nécessité que l'éducation médicale se traduise par des effets tangibles pour l'amélioration de la santé des populations ${ }^{[18]}$. De plus, des analystes de politiques nationales de santé, des groupes spécialisés dans le développement des ressources humaines pour la santé, jusqu'aux Nations-Unies, à travers les objectifs du millénaire pour le développement, ajoutent leurs voix pour appeler à un tel aggiornamento $^{[19,20]}$.

Souhaitons que les différentes agences spécialisées dans l'éducation médicale à travers le monde se concertent pour aboutir à un consensus général sur des normes et des procédures d'évaluation et d'accréditation propres à mieux refléter la responsabilité sociale des institutions de formation et leur impact sur la santé. Il est urgent que cette initiative voie le jour avant que les institutions et les pays ne s'engagent trop avant dans des approches d'accréditation qui n'illustreraient qu'imparfaitement les obligations vis-à-vis de la société.

\section{Tendances d'avenir et raisons d'entreprendre}

Il est probable que nos sociétés, mondialisation aidant, chercheront à justifier leurs investissements à l'aide de données probantes et à promouvoir une régulation pour protéger l'intérêt général face aux dérives de la libre entreprise. Des arguments sur le plan éthique, démocratique, économique et politique en faveur de l'approche CPU sont présentés ci-après.

\section{1- Plan éthique : cause et conséquence}

La tendance à vouloir minimiser les risques pousse nos sociétés à mieux saisir causes et conséquences de toute intervention humaine. Si la pratique des professions de santé est de plus en plus scrutée à l'aune des effets sur l'état de santé des citoyens, il en sera de même pour l'institution de formation. Certes, la recherche d'une corrélation directe entre une stratégie éducationnelle et la santé de la population est incertaine, mais des mesures indirectes sont possibles car on peut distinguer une institution socialement responsable d'une autre qui l'est moins, et démontrer que la responsabilité sociale est garante d'une meilleure adéquation des personnels formés aux besoins prioritaires de la société. 


\section{2- Plan démocratique: ouverture et transparence}

Les domaines désignés naguère comme réservés aux seuls experts deviennent progressivement accessibles au grand public. Le vaste accès à l'information et un esprit critique de plus en plus aigu du citoyen contribuent à un questionnement sur le fonctionnement de toute institution. Le besoin de transparence et la démonstration de l'impact sur la santé, fut-il indirect, s'imposeront progressivement. La reconnaissance explicite d'une institution socialement responsable aura de multiples avantages, entre autres, celui d'éclairer le choix de l'étudiant intéressé à entreprendre une carrière plus conforme à son idéal professionnel et celui d'aider le pouvoir public ou l'autorité de tutelle à mieux identifier l'institution particulièrement disposée à contribuer à des objectifs de développement national.

\section{3- Plan économique : résultats et compétitivité}

Du fait d'une plus grande transparence, comparaison et compétitivité deviendront possibles entre institutions. Quand des normes d'accréditation fondées sur les principes de responsabilité sociale seront disponibles, elles permettront aux pouvoirs publics, aux donateurs et à la société civile de choisir, en meilleure connaissance du potentiel de chaque institution, celle qui mérite les investissements propres à obtenir des résultats à impact social élevé. La propension à démontrer la performance de l'institution par les preuves et la pression en faveur d'une homologation des formations, que suscite la mondialisation, contribueront à une évolution dans ce sens.

\section{4- Plan politique : approche systémique et valorisation des synergies}

La bonne gouvernance de l'institution sera celle qui tiendra compte de la complexité du milieu politique et social ambiant et de l'avantage à créer des partenariats durables avec d'autres institutions du système de santé aux missions similaires ou complémentaires. On peut présumer que le pouvoir politique, soucieux d'efficience et de transparence, sera enclin à attribuer un label d'excellence et un octroi de ressources en fonction de l'aptitude à créer des synergies pour une meilleure cohérence et performance au sein du système de santé.

\section{Conclusion}

Parmi les stratégies possibles pour faire évoluer l'institution de formation des professionnels de santé, celle de l'accréditation est probablement la plus prometteuse car, en même temps qu'elle soutient les États dans leur obligation de régulation pour institutionnaliser une démarche de qualité, elle offre à chaque institution un guide pour conduire son propre développement. Il importe donc qu'on lui accorde une grande attention, en particulier en ajustant l'éventail des normes au regard des principes de responsabilité sociale car il est essentiel que l'institution de formation soit « créditée » pour sa capacité à répondre à l'impérieuse tâche de satisfaire la société dans ses besoins fondamentaux de santé.

\section{Références}

1. Boelen C, Heck J. Définir et mesurer la responsabilité sociale des facultés de médecine, Genève : OMS 2000 [Online]. Disponible sur : http://whqlibdoc. who.int/ hq/1995/WHO_HRH_95.7_fre.pdf

2. Boelen C. Vers l'unité pour la santé. Défi et opportunités des partenariats pour le développement de la santé. Document de travail. Genève : OMS 2002 [Online]. Disponible sur : http://whqlibdoc. who.int/ hq/2001/WHO_EIP_OSD_2000.9_fre.pdf

3. OMS. Médecins pour la santé. Une stratégie mondiale de l'OMS pour la réorientation de l'enseignement de la médecine et de la pratique médicale en faveur de la santé pour tous, Genève : OMS, 1996 ( WHO/HRH/96.1).

4. General Medical Council. Tomorrow's doctors: Recommandations on Undergraduate Medical Education. London: GMC, 2003. [On-line]. Disponible sur : http://www.gmc-uk.org/education/ undergraduate/tomdoc.pdf

5. Frank JR. (Ed). The CanMEDS 2005 physician competency framework. Better standards. Better physicians. Better care. Ottawa: The Royal College of 
Physicians and Surgeons of Canada, 2005. [Online]. Disponible sur : http://crmcc.medical.org/ canmeds/CanMEDS2005/index.php

6. ABIM Foundation, ACP Foundation, European Federation of Internal Medicine. Medical professionalism in the new millennium. A Physician Charter, Ann Intern Med 2002;163 : 243-246. Traduction française : Le professionnalisme médical pour le nouveau millénaire : une charte pour les praticiens. Pédagogie Médicale 2004; 5:43-45.

7. Boelen C. L'éducation médicale vue par un expert international en santé publique. Pédagogie Médicale 2007; 7:844-50.

8. Flexner A. Medical Education in United sates and Canada. The Carnagie Foundation for the Advancement of Teaching 1910; 85.

9. Boelen C. A new paradigm for medical schools a century after Flexner's report. Bull World Health Organ 2002; 80:592-3.

10. Liaison Committee on Medical Education. Functions and structure of a medical school. Standards for accreditation of medical education programs leading to the MD degree 2008 [On-line]. Disponible sur : http://www . $1 \mathrm{cme}$.org/functions2008jun.pdf

11. World Federation for Medical Education. Basic medical education. WFME gobal standards for quality improvement, WFME Office: University of Copenhagen, Denmark 2003 [On-line]. Disponible sur : www.wfme. org

12. Karle H. International recognition of basic medical education programmes, Med Educ 2008;42:12-7.

13. Boelen C, Bandanarayake R, Bouhuijs PAJ, Page GG and Rothman AI. Towards the assessment of quality in medical education. Genève: OMS, 1992 (WHO/HRH/92.7).
14. Gastel BA (Coord.). Toward a global consensus on the quality of medical education: serving the needs of populations and individuals. Acad Med 1995; 70(7):S73-5

15. The Network Towards Unity For Health: Education for Health: Change in Learning and Practice (EfH).[On-line] Disponible sur : http:// www . the-networktufh.org/publications_ resources/educationforhealth.asp

16. Conférence internationale des doyens de facultés de médecine d'expression française. Politique et méthodologie d'évaluation des facultés de médecin et des programmes d'études médicales. CIDMEF, 2006. [On-line]. Disponible sur : http://www.cidmef. $\mathrm{u}$-bordeaux2.fr/

17. Société internationale francophone d'éducation médicale. Facultés de médecine et besoins de société. Vers des nouvelles normes d'évaluation/accréditation. SIFEM : 2007. [On-line]. Disponible sur : http://www.sifem.net/ santeSociete.php

18. Foundation for the advancement of international medical education and research: Improving world health through education. FAIMER [On-line]. Disponible sur : http://www . faimer . org/

19. Global health workforce alliance : Alliance mondiale pour les personnels de santé. Plan stratégique, OMS : 2006. [On-line] Disponible sur : www . who.int/ workforcealliance

20. Nations-Unies. Objectifs du millénaire pour le développement. Rapport 2008. ONU : 2008 [On-line]. Disponible sur : http://www.un.org/french/ millenniumgoals/index.shtml

Correspondance et offprints : Charles Boelen, 585 route d'Excenevex, 74140 Sciez-sur-Léman, France.

Mailto : boelen.charles@wanadoo.fr 